CHERCHER, REPÉRER, AVANCER

\title{
TERRITOIRES : LE MODÈLE « CENTRE-PÉRIPHÉRIE » DÉSUET Gérard-François Dumont
}

L’Esprit du temps | «Outre-Terre»

$2017 / 2 N^{\circ} 51$ | pages 64 à 79

ISSN 1636-3671

ISBN 9782847953978

Article disponible en ligne à l'adresse :

https://www.cairn.info/revue-outre-terre-2017-2-page-64.htm

\section{Pour citer cet article :}

Gérard-François Dumont, «Territoires : le modèle « centre-périphérie » désuet », Outre-Terre 2017/2 (N51), p. 64-79.

DOI 10.3917/oute1.051.0064

Distribution électronique Cairn.info pour L'Esprit du temps.

(C) L’Esprit du temps. Tous droits réservés pour tous pays.

La reproduction ou représentation de cet article, notamment par photocopie, n'est autorisée que dans les limites des conditions générales d'utilisation du site ou, le cas échéant, des conditions générales de la licence souscrite par votre établissement. Toute autre reproduction ou représentation, en tout ou partie, sous quelque forme et de quelque manière que ce soit, est interdite sauf accord préalable et écrit de l'éditeur, en dehors des cas prévus par la législation en vigueur en France. Il est précisé que son stockage dans une base de données est également interdit. 


\section{Territoires : le modèle " centre-périphérie " désuet}

Gérard-François Dumont' ${ }^{1}$

Depuis les prémices de la littérature sur les questions territoriales, le modèle centre-périphérie est prédominant et le concept de centre est considéré comme un des plus importants en économie spatiale et en géographie. Il signifie que les territoires s'organiseraient systématiquement de façon hiérarchique selon une dualité entre un centre dominant et des périphéries dominées, le centre disposant d'une concentration de population, d'activités économiques, d'offre culturelle et de lieux de pouvoir. Le centre, avec sa position privilégiée, serait le maitre et le régulateur de territoires situés à sa périphérie.

Au fil des derniers siècles, les présentations du modèle centre-périphérie ont varié, mais sans jamais véritablement le remettre en cause. Les structures territoriales, inévitablement alignées sur une logique centre-périphérie, se caractériseraient donc par un centre totalement polarisant et, en conséquence, une asymétrie et des inégalités entre le centre et ses périphéries. Dans une première partie, nous analyserons les éléments montrant combien le modèle centre-périphérie continue d'être prégnant. Pourtant, ce modèle conserve-t-il au XXI ${ }^{\mathrm{e}}$ siècle une indépassable véracité? Répondre à cette question, c'est analyser, dans une seconde partie, les réalités territoriales qui conduisent à se demander si le modèle centre-périphérie n’est pas désuet.

\section{LE MODELE CENTRE-PÉRIPHÉRIE PRÉDOMINANT...}

Pour illustrer combien le modèle centre-périphérie est prédominant, il faut considérer la littérature, les concepts statistiques et les réglementations. Dans la littérature, les principaux auteurs en ont fait une présentation quasi systématique en dépit de nuances liées à leurs propres expériences ; il est ainsi possible de présenter des travaux allant de Richard Cantillon au XVIII' siècle à ceux du prix Nobel d'économie 2008, Paul Krugman.

1 Professeur à I'Université de Paris-Sorbonne, Président de la revue Population \& Avenir <www.population-demographie.org/revue03.htm > 
Dès Richard Cantillon (1680-1734), dont la pensée peut se définir comme un mercantilisme critique, le modèle centre-périphérie est fécondé dans son livre Essai sur la nature du commerce en général (publié à titre posthume en 1755). Richard Cantillon est un banquier ayant voyagé dans toute l'Europe, le Japon et le Brésil à une époque où la production économique est très majoritairement issue de l'agriculture. Selon lui, l'existence de villes, donc de centres, tient à la localisation de la puissance publique, des seigneurs et des grands propriétaires, qui tirent leurs revenus de l'agriculture. La richesse de la ville vient de l'exploitation de son environnement rural, notamment par les revenus des propriétaires fonciers et les activités des marchés de produits. Quant aux industries, elles sont localisées dans les villes pour optimiser les coûts des transports. L'organisation du territoire s'effectue à partir des villes selon une logique hiérarchique qu'elles dominent. Le niveau inférieur aux villes est celui des bourgs, gros villages où s'échangent des produits, ceux de l'agriculture contre ceux de l'artisanat et de l'industrie. À l'échelon moindre se trouvent les villages. Leur localisation tient au temps de déplacement entre le lieu d'habitation et le lieu de travail des agriculteurs, donc les champs.

Certes, pour Cantillon, c'est bien le périphérique qui produit l'essentiel des richesses, mais celles-ci sont essentiellement captées par le centre, ce qu'il déplore. Il souhaiterait que les industries de transformation des produits agricoles soient également installées dans le monde rural, ce qui inciterait au développement d'une économie des transports.

Au début du XIX ${ }^{\mathrm{e}}$ siècle, Johann Heinrich von Thünen (1783-1850) propose en $1826^{3}$ une analyse selon laquelle la ville, en tant que centre, se trouve être l'organisatrice des espaces périphériques où s'effectuent les productions agricoles. Von Thünen part d'observations conduites dans la région de Rostock (Mecklembourg) où il exploite un domaine agricole de 340 hectares. Il considère que les frais de transport des productions agricoles sont fonction du poids des produits et de la distance à parcourir pour rejoindre la ville. Sous cette hypothèse simplificatrice, von Thünen différencie six couronnes territoriales entourant la ville devant pratiquer des cultures différentes, allant de la zone de maraîchage (dont les produits doivent être amenés frais en ville chaque jour) à la zone d'élevage extensif (où il faut des mois pour élever et engraisser les animaux). Ainsi, la fonction économique de la ville, avec les

2 Réédition INED, Paris, 1952.

3 Johann Heinrich von Thünen, Der isolierte Staat in Beziehung auf Landwirtschaft und Nationalökonomie, Hambourg et Rostock, 1826, 1842 et 1850 ; traduction française partielle : L'État isolé, Laverrière, Paris, 1851 et 1857. 
besoins variés des citadins, commande la géographie des périphéries où s'effectuent les productions agricoles ${ }^{4}$.

Certes, von Thünen montre également comment des infrastructures nouvelles, comme la mise en état de navigation d'une voie d'eau, peuvent, à une époque où les transports par voie d'eau sont estimés dix fois moins coûteux que les transports terrestres, modifier l'utilisation économique des espaces périphériques. Mais il maintient sa théorie des "cercles concentriques " imposés par la ville qui se trouve au centres.

Au début du XX $\mathrm{XX}^{\mathrm{e}}$ siècle, il convient de considérer les activités industrielles dont l'importance s'est considérablement accrue. D'où les travaux, en 1909, d'Alfred Weber (1868-1958) ${ }^{6}$, frère cadet du sociologue Max Weber, s'interrogeant, selon une approche plutôt microéconomique, sur les stratégies de localisation des entreprises industrielles. Selon Alfred Weber, les coûts de transport sont une partie essentielle des prix de revient. Le coût de la distance rend donc stratégique le choix de la localisation qui dépend, d'une part, de la distance avec les fournisseurs des matières premières pour l'entreprise de production et, d'autre part, de celle avec les distributeurs ou les consommateurs des produits fabriqués. Alfred Weber insiste sur un deuxième élément du prix de revient, la main-d'œuvre, dont le coût doit être supportable pour l'entreprise, ce qui suppose selon lui un large marché du travail. Il introduit un troisième élément, le fait que les économies résultant de la production de masse peuvent inciter les industries à se localiser dans des agglomérations. Il énonce donc une " force agglomératrice " dont la puissance est plus ou moins grande, et qui doit être relativisée en fonction des coûts fonciers éventuellement plus élevés. Mais il considère que l'installation de plusieurs entreprises dans un même lieu entraîne des avantages pour la production et la commercialisation.

Certes, Alfred Weber note l'existence possible de facteurs de " déglomération ", ceux qui augmentent les coûts de production, dont le principal est lié aux coûts fonciers, la concentration rendant rare les emplacements disponibles, ce qui en augmente le prix. Mais son analyse pousse à la concentration, donc à privilégier le centre qui optimiserait le prix de revient des activités productives.

\footnotetext{
4 II conviendrait en réalité de prendre également en compte la géographie physique. Les villes sont souvent situées dans les vallées, là où se trouvent les terres grasses et humides nécessaires aux cultures maraîchères, alors que la culture des céréales et l'élevage extensif se pratiquent sur les plateaux secs.

5 Cf. également Claude Ponsard, Histoire des théories économiques spatiales, Armand Colin, Paris, 1958.

6 Alfred Weber, Uber den Standort der Industrien, Tübingen, J.C.B. Mohr (Paul Siebeck), 1909 ; traduction américaine Theory of the location of industries, University of Chicago Press, Chicago, $2^{e}$ éd., 1957.
} 
Une vingtaine d'années plus tard, dans des textes de 1929 et $1931^{7}$, le modèle centre-périphérie se trouve à nouveau privilégié avec la loi de William Reilly, appelée également le modèle gravitaire, et qui se résume ainsi : « une ville attire la clientèle en raison directe de sa population agglomérée et en raison inverse du carré de la distance $»^{8}$. Cette loi, inspirée par la loi de la gravitation universelle d'Isaac Newton, sans pouvoir atteindre à la rigueur mathématique de cette dernière, débouche sur une quantification des aires périphériques dominées par l'influence des villes.

Pratiquement à la même époque, le modèle centre-périphérie se trouve promu par l'étude de la géographie du commerce de Walter Christaller dont les travaux présentent une " théorie des places centrales ". Selon cet auteur, la ville est le lieu où se regroupent des commerces ayant une aire de marché de dimension identique ou voisine. Pour Christaller, c'est l'organisation efficiente du commerce qui explique le rôle central d'une ville dont l'importance, c'est-à-dire l'étendue des périphéries qu'elle domine, dépend de trois ensembles d'éléments : les premiers sont le volume de la population desservie et les économies d'échelle possibles qui décident de la qualité et du coût du service commercial ; les deuxièmes sont liés à la distance qui influence les coûts ; les troisièmes sont corrélés avec la fréquence des achats des produits qui appellent une plus ou moins grande proximité. W. Christaller définit alors la "portée » d'un lieu (ou d'un centre), c'est-à-dire la distance que le consommateur accepte de parcourir pour acquérir un bien. Cette portée est notamment fonction de la valeur du bien acquis. Les différentes aires de marché génèrent des villes de taille différentes et donc une hiérarchie commerciale des territoires.

Avec August Lösch ${ }^{10}$, l'analyse se recentre sur la géographie des industries, explicitée par un système d'équations assez complet débouchant sur la " théorie des lieux centraux ». Les productions industrielles sont les facteurs explicatifs de la concentration urbaine. L'espace a une organisation dichotomique : aux villes les implantations industrielles et les marchés des biens, aux autres territoires les productions et les marchés agricoles selon une distribution spatialement uniforme. Les raisons explicatives de la concentration des industries dans les villes sont les économies d'agglomération et la minimisation des coûts de transport globaux. Les économies d'agglomération, moteurs de la formation des villes, s'expliquent par quatre séries de facteurs:

\footnotetext{
7 William J. Reilly, "Methods of the study of retail relationships ", University of Texas Bulletin, n²944, novembre 1929, et The Law of Retail Gravitation, New York, G. P. Putnam's Sons, 1931.

8 Cf. André Piatier, Radioscopie des communes de France, Paris, Economica, 1979, p. 44.

9 Walter Christaller, Die zentralen Orte in Suddeutschland, léna, G. Fisher, 1933 ; traduction américaine Central places in Southern Germany, Englewood Cliffs (N.J.), Prentice Hall, 1956.

10 August Lösch, "The Nature of Economic Regions ", Southern Economic Journal, vol. 5, nº 1, juillet 1938 et Die raümliche Ordnung der Wirtschaft., léna, G. Fisher, 1940 (2éd. 1944), 380 p. ; traduction américaine The Economics of Location, New Yaven, Yale University Press, 1954.
} 
"-les avantages de la production en grand (de masse) peuvent entraîner la concentration en un lieu de grandes entreprises individuelles;

- des entreprises de même type peuvent être portées à s'agglomérer par l'attrait des économies externes (environnement moins cher, partage de coûts), par les avantages techniques du site, par des possibilités plus grandes de concurrence ;

- des entreprises hétérogènes peuvent se rapprocher en raison de leurs liens d'interdépendance (entreprises et sous-traitants, flux tendus, services aux entreprises) ;

- enfin des villes peuvent naître de la seule agglomération de consommateurs ${ }^{11}$.

Les travaux d'August Lösch conduisent à proposer une justification économique de l'existence de centralités urbaines avec la théorie des aires de marché. En effet, en considérant par hypothèse un espace homogène au départ, homogène tant en ce qui concerne les ressources que les hommes (densité, revenus, préférences), mais en prenant en compte des agents économiques qui cherchent à optimiser leurs avantages, Lösch arrive à la conclusion que cet espace va devenir hétérogène. Autrement dit, des phénomènes urbains vont apparaitre. C'est le résultat d'un facteur technique, la concentration qui crée des économies d'échelle, et d'un facteur géographique, la dispersion ou la distance qui entraîne des coûts de transport.

Au début du XXIe siècle, le modèle centre-périphérie est à nouveau honoré avec la remise du prix Nobel d'économie à Paul Krugman, le 13 octobre 2008, notamment pour ses travaux sur la localisation de l'activité économique ${ }^{12}$. À en croire l'analyse, que l'on range sous la dénomination de "nouvelle économie géographique ", de ce prix Nobel, diverses raisons poussent l'activité économique à se concentrer sur un nombre limité de villes qui se trouvent être attractives. Selon le "modèle centrepériphérie, les industries se localisent dans un lieu en tenant compte de l'arbitrage entre les économies d'échelle, qui favorisent la concentration, et les coûts de transport, qui favorisent la dispersion. Chaque industrie tente alors de desservir son marché en minimisant les coûts de transport, c'est-à-dire en se rapprochant de la demande locale.

Cela entraîne un processus circulaire : les industries recherchent des localisations où la demande locale est forte tandis que la demande locale est d'autant plus forte que de nombreuses industries ont choisi cette localisation. Ce processus est à l'avantage des grands marchés. Pour expliquer les concentrations géographiques particulièrement importantes d'industries manufacturières ou de services, Krugman s'appuie sur les externalités marshalliennes classiques. Les avantages liés à la taille des marchés sont ren-

11 Cf. Joseph Lajugie, Pierre Delfaud, Claude Lacour, Espace régional et aménagement du territoire, Paris, Dalloz, 1985, p. 53.

12 Ainsi que pour son analyse des modèles du commerce mondial. 
forcés dans certains lieux par des externalités technologiques ou informationnelles liées à l'importance de la proximité dans la transmission du savoir et des connaissances " 13 .

Ainsi, l'importance donnée au modèle centre-périphérie, au fil des derniers siècles, est susceptible d'expliquer son influence, explicite ou non, sur des concepts statistiques comme sur des décisions politiques.

\section{... DANS DES CONCEPTS STATISTIQUES}

Considérons le cas de la France. Il est incontestable que des critères extensifs ont été et sont utilisés pour définir l'aire des périphéries supposées dominées par des centres. En effet, ce que l'Insee désigne comme " unité urbaine » est un espace statistique fondé sur un postulat selon lequel des territoires considérés en continuité de cadre bâti fonctionnent selon une logique centre-périphérie engendrant un espace spécifique unitaire ${ }^{14}$. L'hypothèse implicite est que cette continuité signifierait automatiquement que les territoires périphériques situés dans une unité urbaine sont dominés par un centre, correspondant souvent à la commune géographiquement centrale ou la plus peuplée. ET le concept d'unité urbaine utilise une définition extensive : il y aurait continuité dès que l'écart entre deux bâtiments est de 200 mètres ${ }^{15}$. Ce critère quantitatif, affirmé et non justifié, engendre des périmètres sur lesquels toute discussion est quasiment impossible, comme si la définition de l'unité urbaine était scientifiquement indiscutable alors qu'elle résulte d'un choix s'appuyant sur le modèle centre-périphérie. L'histoire des délimitations des unités urbaines montre l'extension de nombre d'entre elles au fil des recensements, sans apporter la preuve que l'ensemble des territoires appartenant à une unité urbaine concernée aient un fonctionnement partagé, gouverné du centre. En outre, en 2010, pour fixer le périmètre des territoires urbains, l'Insee a posé une définition encore plus extensive de la règle des 200 mètres en dessous de laquelle le cadre bâti est jugé continu' ${ }^{16}$.

Le caractère extensif des concepts choisis par l'Insee s'est trouvé particulièrement mis en évidence dans les années 1960, avec celui de «zone de peuplement industriel

13 Cf. Olivier Walther, "Paul Krugman: (presque) un Nobel de géographie ", Articulo, 2008, cité in Gérard-François Dumont, Laurent Chalard, "L'attractivité des territoires : théories et réalités ", Population \& Avenir, n697, mars-avril 2010. 14 Cf. Gérard-François Dumont (dir.), La France en villes, Paris, Sedes, 2010.

15 L'application de la règle des 200 mètres ignore des coupures territoriales comme les cours d'eau en l'absence de ponts, gravières, dénivelés importants. Depuis le découpage Insee de 2010, certains espaces publics (cimetières, stades, aérodromes, parcs de stationnement...), terrains industriels ou commerciaux (usines, zones d'activités, centres commerciaux...), ont été traités comme des bâtis avec la règle des 200 mètres pour relier des zones de construction habitées, à la différence des découpages précédents où ces espaces étaient seulement annulés dans le calcul des distances entre bâtis.

16 Par exemple, ces normes statistiques Insee ont conduit à la fusion des unités urbaines de Lyon et de Villefranche-surSaône lors de la nouvelle délimitation 2010, alors que la morphologie du territoire existant entre ces deux unités urbaines, qui étaient séparées selon les délimitations antérieures, a surtout un caractère rural, comme les automobilistes qui empruntent l'autoroute A6 le constatent aisément. 
ou urbain » (ZPIU). Il s'agissait d'élargir le champ de domination du centre au-delà des périphéries déjà comprises dans l'unité urbaine à des territoires relativement éloignés. Ces périphéries comprenaient des zones d'activités consacrées au commerce, au tertiaire ou à la logistique, ou des zones résidentielles. Puis, à la fin des années 1980, la délimitation en ZPIU se révèle obsolète. En effet, après la publication des chiffres du recensement de 1990, 96\% de la population de la France se trouvent en ZPIU, ce qui signifie que le zonage en ZPIU ne permet plus de distinctions territoriales pertinentes entre les territoires en ZPIU et ceux qui ne le sont pas.

Après l'abandon du concept de ZPIU, le nouveau concept utilisé par l'Insee, celui d'aire urbaine, suit une même logique radiale, avec des critères considérés comme rationnels et donc légitimes alors qu'ils sont purement subjectifs. D’abord, le périmètre minimum d'une aire urbaine est fondé sur la taille de l'unité urbaine, donc sur une taille minimum discutable. Ensuite, ce concept d'aire urbaine élargit l'unité urbaine en lui associant d'autres territoires qu'il considère également commandés du centre, sous prétexte d'une règle mathématique selon laquelle au moins $40 \%$ de la population résidente ayant un emploi travaille dans le périmètre de l'unité urbaine ou dans des communes attirées par celle-ci. Ce niveau de $40 \%$ n'est nullement justifié, ce qui signifie qu'un autre pourcentage pourrait modifier considérablement la superficie de l'aire urbaine et l'importance des espaces situés hors des aires urbaines, les territoires à dominante rurale ${ }^{17}$.

La prégnance du modèle centre-périphérie transparait non seulement dans les théories ou dans les concepts statistiques, mais elle se décline aussi dans des réglementations, tout particulièrement en France.

\section{... DANS DES RÉGLEMENTATIONS}

De façon générale, les réglementations françaises visent à favoriser les centres au détriment des périphéries. Ceci peut être illustré par un exemple qui revient annuellement, celui de la répartition de la dotation globale de fonctionnement par habitant versée par l'État aux communes; elle est proportionnelle à la taille de la commune. Les communes ayant plus de 200000 habitants perçoivent la dotation maximale. Ensuite, le montant est dégressif jusqu'aux communes de moins de 500 habitants dont la dotation par habitant est deux fois moindre. De nombreux financements de l'État mettent en évidence cette volonté de privilégier le centre.

17 Cf. Gérard-François Dumont, "Pour analyser la ruralité, balayer les paradigmes dominants ", Cahiers de la fonction publique, $n^{\circ} 356$, juin 2015. 
Plus récemment, les quatre principales lois territoriales ${ }^{18}$ votées pendant le quinquennat de François Hollande, comme précédemment d'autres lois votées sous les quinquennats précédents, se réfèrent, plus ou moins explicitement, au modèle centre-périphérie. La création systématique de grandes régions, décidées en violant un traité international ${ }^{19}$, a pour effet de donner plus de poids à un centre - la capitale régionale - en augmentant la superficie de ses périphéries et en y concentrant les lieux de décision. Les lois créant les métropoles ${ }^{20}$, qui utilisent le concept d'aire urbaine présenté ci-dessus comme mesure du modèle centre-périphérie, ont pour effet de donner davantage de pouvoir aux centres tant par rapport aux périphéries incluses dans le périmètre des territoires désormais appelés métropoles que par rapport aux périphéries situées au delà de ces périmètres. La loi sur la nouvelle organisation territoriale de la République (NOTRe) contraint les périphéries à abandonner des compétences et à les transférer au centre et ce, de façon uniforme sur les territoires français, sans tenir compte des spécificités géographiques, historiques ou culturelles des territoires, ni de choix qui auraient pu être possibles en matière de gouvernance territoriale ${ }^{21}$.

Dans la nouvelle cartographie des intercommunalités mise en place en 2017, l'application quasi systématique du principe "big is beautiful » sans tenir compte ni du vécu des populations ${ }^{22}$, ni de la géographie des territoires traduit l'idée selon laquelle le centre est dominateur. Par exemple, Poitiers se trouve désormais, donc depuis 2017, dominant administrativement une vaste périphérie, dont les territoires du Futuroscope ${ }^{23}$ qui ne lui doivent rien $^{24}$ et qui étaient très faiblement en interaction avec le chef-lieu du département de la Vienne, comme le montrait leur indice très élevé de concentration d'emploi, soit leur rapport entre le nombre d'emplois offerts sur les territoire du Futuroscope et ses actifs ayant un emploi qui y résident (plus de $80 \%$ ) ou l'analyse géographique ${ }^{25}$.

18 Loi du 27 janvier 2014 de modernisation de l'action publique territoriale et d'affirmation des métropoles (MAPTAM) ; loi du 17 janvier 2015 relative à la délimitation des régions, aux élections régionales et départementales et modifiant le calendrier électoral ; loi du 7 août 2015 sur la nouvelle organisation territoriale de la République (NOTRe) ; et la loi du 28 février 2017 relative au statut de Paris et à l'aménagement métropolitain.

19 En effet, la Charte européenne de l'autonomie locale précise, dans son article 5, que «pour toute modification des limites territoriales locales, les collectivités locales concernées doivent être consultées préalablement, éventuellement par voie de référendum là où la loi le permet ». Et le Congrès des pouvoirs locaux et régionaux du Conseil de l'Europe a fini par dénoncer cette violation, voir " La démocratie locale et régionale en France ", mars 2016, point 208.

20 Statut de métropole créé sous forme d'établissement public de coopération intercommunale par la loi du 16 décembre 2010, puis élargie par la loi du 27 janvier 2014 de modernisation de l'action publique territoriale et d'affirmation des métropoles (MAPTAM), à nouveau élargie par la loi du 28 février 2017 relatif au statut de Paris et à l'aménagement métropolitain.

21 Cf. Gérard-François Dumont, "Favoriser une meilleure gouvernance des territoires ", in Joël Allain, Philippe Goldman, Jean-Pierre Saulnier, De la prospective à l'action, Bourges, Apors Éditions, 2016.

22 Cf. Jean-Marc Callois, "Le citoyen, grand oublié des réformes territoriales ", Population \& Avenir, n732, mars-avril 2017.

23 Par Futuroscope, il faut entendre non seulement la partie la plus connue, le parc du Futuroscope, parc de loisirs à thème technologique, scientifique, d'anticipation et ludique, dont les attractions mélangent approches sensorielles et projections d'images, mais l'ensemble des activité économiques créées.

24 En réalité, Poitiers, comme de nombreux acteurs politiques des années 1980, croyaient à l'échec du projet Futuroscope.

25 Cf. Jacques Beauchard, "La ville contre le territoire? », Population \& Avenir, n708, mai-juin 2012. 
Autrement dit, les différentes lois territoriales françaises votées depuis les années 2010 peuvent se résumer à deux idées : premièrement, " big is beautiful " ou plus un centre est de grande taille, plus son rayonnement crée de richesses; en second lieu, les territoires fonctionnent selon une logique radiale, où des périphéries sont commandées et dépendantes du centre qui les tient attachées par les rayons qu'il déploie.

Ces deux idées conduisent au postulat suivant : plus les rayons administratifs sont longs, plus le centre influence un territoire vaste et plus l'ensemble réunissant le centre et des périphéries dans une institution unique, disposant d'un maximum de pouvoirs, sera puissant.

En conséquence, puisque les territoires sont censés fonctionner ainsi, les lois territoriales françaises ont développé des organisations territoriales exerçant des fonctions politiques et administratives sur un vaste espace, et en les organisant de la façon la plus centralisée possible. Selon cette logique radiale, d'une part, la localisation des activités et des habitants dans les périphéries ne pourrait s'expliquer qu'en raison de l'existence d'un centre et, d'autre part, les activités et les habitants des périphéries ne pourraient satisfaire leurs besoins qu'en recourant au centre.

Mais cette prégnance théorique, statistique et réglementaire du modèle centrepériphérie est-elle pleinement justifiée?

\section{LE MODĖLE CENTRE-PÉRIPHÉRIE DÉSUET ?}

Si le modèle centre-périphérie conservait toute sa pertinence, la vie des périphéries dépendrait essentiellement du rayonnement du centre. Or, de nombreux exemples montrent que des réussites territoriales sur des espaces périphériques ne doivent absolument rien à un quelconque centre. En outre, l'analyse fine des évolutions territoriales montre une importance croissante de la nature réticulaire des territoires alors que le modèle centre-périphérie les fige dans une logique exclusivement radiale. En conséquence, il importe de décliner les raisons qui donnent à ce modèle centre-périphérie un caractère désuet.

\section{DeS RÉUSSITES TERRITORIALES INEXPLICABLES PAR LE MODĖLE CENTRE-PÉRIPHÉRIE}

Examinons des réussites territoriales de ces dernières décennies en France. Doivent-elles systématiquement à l'existence de centres qui les auraient fécondées 
grâce à leur rayonnement ? La réponse à cette question est négative, car nombre de réussites reposent plutôt sur l'innovation endogène, selon une logique d'entrepreneuriat, sans aucun facteur lié à une éventuelle irrigation d'un lieu périphérique par un centre. Citons quelques exemples. En Bretagne, la réussite des vêtements Saint James ne doit rien au centre régional qu'est Rennes. En Normandie, la réussite à Villedieu-les-Poêles de Mauviel, entreprise qui vend des ustensiles de cuisine en cuivre dans le monde entier, notamment à des cuisiniers étoilés, ne doit rien à un centre comme l'ancienne capitale régionale Caen. En Charente-Maritime, le succès des produits "Eau thermale Jonzac ", permis par la création d'une station thermale en 1986, ne doit rien ni au centre du département, La Rochelle, ni à la capitale régionale, Poitiers, non plus qu'à la métropole la plus proche, Bordeaux. Dans la Haute-Loire, le renouveau du village enclavé de Saint-Bonnet-le-Froid, situé à $1117 \mathrm{~m}$ d'altitude, ne doit rien à l'influence d'un quelconque centre, qu'il s'agisse du chef-lieu du département Le Puy-en-Velay, de la ville plus peuplée Saint-Étienne ou de la métropole la plus proche, Lyon. Dans les Pays de la Loire, les succès de Bénéteau, champion mondial des bateaux de plaisance, ou de Fleury-Michon, numéro 1 français du jambon, ne doivent rien à la "métropole » de Nantes ${ }^{26}$. En Midi-Pyrénées, le développement de l'industrie pharmaceutique à partir de Castres, avec les Laboratoires Pierre Fabre ${ }^{27}$ créés en 1962, ne doit rien au centre qu'est Toulouse, la capitale régionale. Bien au contraire, c'est la métropole toulousaine qui, depuis, bénéficie de cette réussite, par exemple avec l'ouverture, en 2010, du centre de $\mathrm{R} \& \mathrm{D}^{28}$ de Fabre sur le campus de l'Oncopole à Langlade (Toulouse). En Auvergne, la réussite de Michelin ou de Limagrain, quatrième producteur de semences dans le monde, ne peut s'expliquer par la présence d'un centre, Clermont-Ferrand, capitale régionale jusqu'en 2015, mais seulement par l'innovation constante chez ce fabriquant de pneumatiques. En Limousin, les succès de Legrand, leader mondial des produits et systèmes pour installations électriques, n'ont aucun lien avec un quelconque centre métropolitain.

Ces exemples attestent que des territoires vus comme des périphéries peuvent présenter des réussites sans aucune possibilité de les expliquer par l'existence d'un centre qui aurait rayonné sur eux. Ils démentent le modèle centre-périphérie qui, en outre, se voit dépassé par la nature de plus en plus réticulaire des territoires.

26 Plus généralement, l'automaticité d'attractivité des métropoles, souvent avancée, n'est nullement démontrée, cf. Gilles Poupard, "Développement local et emploi productif : un monopole des métropoles ? ", Population \& Avenir, n725, novembre-décembre 2015 ; Jean-Marc Zaninetti, "La crise et l'attractivité des villes : Paris en recul ", Population \& Avenir, n730, novembre-décembre 2016.

27 Cf. Hervé Penan, Histoire des Laboratoires Pierre Fabre, Toulouse, Éditions Privat, 2014.

28 Recherche et développement. 


\section{LE MODĖLE CENTRE-PÉRIPHÉRIE EST RADIAL, LA RÉALITÉ TERRITORIALE DE PLUS EN PLUS RÉTICULAIRE}

En effet, les concepts d'unité urbaine et d'aire urbaine font un fort effet de loupe en grossissant les soi-disant impacts du modèle centre-périphérie et en exagérant la portée géographique de ce modèle puisque les critères retenus par ces concepts s'exercent de façon cumulative ${ }^{29}$. Or ils sont inappropriés. En effet, ils peuvent donner l'impression que le territoire formant une unité urbaine ou une aire urbaine fait système, c'est-à-dire réunit un ensemble d'éléments qui se trouvent en interaction, au sein d'un périmètre géographique qui ferait unité. Selon cette logique radiale, d'une part, la localisation des activités et des habitants dans les périphéries ne pourrait s'expliquer qu'en raison de l'existence d'un centre et, d'autre part, les activités et les habitants des périphéries ne pourraient satisfaire l'ensemble de leurs besoins qu'en recourant au centre.

Or, deux raisons expliquent que ce n'est nullement le cas. En premier lieu, y compris au sein de vastes unités urbaines, c'est souvent la proximité qui domine. Pour prendre l'exemple de Paris, des communes de l'ouest de l'unité urbaine de Paris, comme Mantes-la-Jolie, ne font nullement système avec des communes du nord, de l'est ou du sud de Paris et pas nécessairement avec Paris. En effet, 40\% des actifs d'Île-de-France travaillent dans leur commune d'habitation ou dans une commune limitrophe. Cette proportion monte à $60 \%$ si le rayon est de deux communes. Ainsi les territoires enfermés dans les concepts d'unité urbaine ou d'aire urbaine sont souvent des juxtapositions de microcosmes assez hermétiques. Donc, tout particulièrement dans les vastes unités urbaines, les territoires qui les composent ne vivent guère, ou pas, en symbiose et l'unité urbaine ne mérite nullement ce nom d'unité.

En second lieu, les territoires fonctionnent de façon réticulaire et non radiale. Le fonctionnement supposé radial qui dicte la construction statistique de territoires désignés comme unités urbaines et aires urbaines, et devenus en conséquence depuis 2017 de vastes unités administratives sous le nom de métropoles ou de communautés d'agglomération, conduit inévitablement à ignorer que de nombreux habitants qui résident dans l'un des territoires d'une unité urbaine ou d'une aire urbaine ignorent parfois totalement la commune géographiquement située au centre, ou n'y ont recours que de façon exceptionnelle. Pour de nombreux habitants, l'espace vécu, pour leurs activités professionnelles, pour leur consommation et pour leurs loisirs, ne correspond nullement à une logique radiale. Au contraire, il s'inscrit dans un réseau de territoires qui, d'une part, ne recoupe nullement le périmètre de l'unité

29 Cf. Gérard-François Dumont, Laurent Chalard, " Pour une nouvelle analyse territoriale ", in Gabriel Wackermann, (dir.), L'écosociété, Paris, Éditions Ellipses, février 2010. 
urbaine ou de l'aire urbaine et, d'autre part, peut ignorer la commune-centre. En conséquence, à l'heure des mobilités, le fonctionnement des territoires ne peut se réduire à une logique centre-périphérie, selon laquelle tout partirait du centre et reviendrait au centre, mais correspond à une logique réticulaire.

Effectivement, de nombreux territoires fonctionnent selon des mobilités domicile-travail, domicile-lieux de consommation, domicile-lieux de loisirs, domicilelieux des activités sociales/associatives, amicales ou avec la famille élargie, qui se passent totalement du centre. Pour de nombreux habitants et activités, les mobilités ne s'inscrivent nullement dans les logiques radiales sur lesquelles se fondent non seulement les mesures statiques des unités urbaines et des aires urbaines, mais aussi plusieurs lois territoriales récentes ${ }^{30}$. Nombre d'habitants vivent les territoires selon des logiques réticulaires, logiques d'ailleurs facilitées par les technologies de l'information et de la communication (TIC) dont les logiciels de navigation désormais à la disposition des très nombreux possesseurs de Smartphones.

L'« unité » urbaine n'est alors qu'une addition de territoires dont la vie ne s'inscrit ni dans une logique systémique, ni dans une règle de complémentarité, non plus que dans une hiérarchie centre-périphéries.

\section{QUATRE RAISONS DE LA DÉSUÉTUDE DU MODĖLE CENTRE-PÉRIPHÉRIE}

Au total, le modèle centre-périphérie s'avère désuet pour quatre raisons. Première raison, nombre des territoires qui sont considérés comme des centres et qui, souvent, ne se voient que centraux sont aussi en situation périphérique. Donnons l'exemple de Paris, la capitale française, celle jugée par Madelon ${ }^{31}$ comme « le grand bureau des merveilles, le centre du bon goût, du bel esprit ». Bien sûr, Paris est un centre : la majorité des sièges sociaux des plus grandes entreprises françaises sont localisées à Paris. Pourtant, Paris est aussi en situation périphérique : périphérie de décisions prises à Bruxelles dans le cadre de l'Union européenne ; périphérie de décisions prises à l'OMC dans le cadre de décisions commerciales internationales ; périphérie par rapport aux sièges sociaux des grandes entreprises non européennes. Ces dernières peuvent par exemple se demander où, en Europe, elles vont installer ou maintenir leur siège social européen : à Paris, à Londres, à Munich, à Francfort, à Milan... ? Ainsi, même Paris dont on pourrait penser, compte tenu d'un raisonnement jacobin, que c'est exclusivement une ville centrale, est devenu aussi une ville

30 Cf. Gérard-François Dumont, "Territoires : un fonctionnement radial ou réticulaire ? ", Population \& Avenir, n723, mai-juin 2015.

31 Poquelin, Jean-Baptiste, dit Molière, Les précieuses ridicules, scène X, 1659. 
périphérique, comme l'atteste la dépendance de ses activités financières vis-à-vis de Londres tant avant le Brexit que pour essayer de profiter des effets de relocalisation à partir de Londres dus au Brexit ${ }^{32}$.

Deuxième raison, les territoires considérés a priori comme des périphéries d'un seul centre sont, de plus en plus souvent, non pas en situation de périphérie par rapport à un unique territoire qui les dominerait, mais se révèlent pluripériphériques. Donnons un exemple. Un regard rapide sur une carte par points de la géographie du peuplement de la moitié ouest du département des Pyrénées-Atlantiques peut donner l'impression que la commune d'Espelette n'est qu'une périphérie de Bayonne ; or, ce n'est nullement le cas. Il est vrai que cette commune d'Espelette, pour bénéficier de certains services publics localisés à Bayonne en matière d'éducation, de santé ou de besoins administratifs, se trouve en situation de dépendance avec Bayonne, une sous-préfecture à fonctions élargies et, depuis le $1^{\text {er }}$ janvier 2017, capitale démographique de la communauté d'agglomération du Pays basque. La dualité Bayonne-Espelette semble alors confirmer le modèle centrepériphérie. Mais, avec l'extraordinaire renaissance du piment d'Espelette ${ }^{33}$, due à de l'innovation purement locale et nullement au rayonnement d'un quelconque centre, cette commune est beaucoup plus dépendante des acquéreurs de son piment et de sa notoriété qui attire des touristes du monde entier. Espelette est par exemple dépendante des grandes centrales d'achat qui décident - ou non - de commander son piment, des restaurateurs établis dans le monde entier qui valorisent - ou non - l'utilisation de ce piment dans leur cuisine, de territoires qui choisissent - ou non - de s'associer au piment d'Espelette dans leur production, comme le sel de Guérande. Autre exemple, un raisonnement semblable pourrait être tenu en considérant la commune de Vitré au regard de la capitale régionale Rennes ${ }^{34}$.

Plus généralement, le caractère pluripériphérique des territoires est un phénomène général : prenons l'exemple de Nice. Bien que chef-lieu des Alpes-Maritimes, cette ville est aussi périphérique, déjà au titre de différentes décisions politiques, de Marseille où siègent le Conseil Régional Provence-Alpes-Côte d'Azur et la préfecture de région, ou de Paris où gouvernements et Parlement prennent des décisions qui la concernent. En même temps, son industrie touristique fait de Nice une périphérie de Moscou, parce qu'une partie significative de l'activité touristique de la Côte d'Azur, depuis les années 1990, est la conséquence de l'implosion soviétique et du retour des Russes sur la Côte d'Azur après sept décennies. En

32 Cf. Gérard-François Dumont, Pierre Verluise, Géopolitique de l'Europe : de I'Atlantique à I'Oural, Paris, PUF, 2016.

33 Cf. Gérard-François Dumont, "La France des marges et l'indispensable attractivité des territoires ", in Raymond Woessner, La France des marges, Paris, Atlande, 2016.

34 Cf. Gérard-François Dumont, Diagnostic et gouvernance des territoires, Paris, Armand Colin, 2012 et « Villes à système productif industriel et gouvernance territoriale ", Bulletin de l'Association de Géographes Français (AGF), vol. 92, décembre 2015. 
outre, la région niçoise est également une périphérie des États-Unis puisque de nombreuses entreprises américaines y ont installé des établissements et parfois leur siège social européen, notamment sur la technopole de Sophia Antipolis. Suivant cet exemple, les territoires doivent donc penser leur développement en prenant en compte leur caractère pluripériphérique, fort différent de l'approche bipolaire du modèle centre-périphérie.

Troisième raison de mise en cause de ce modèle, des territoires dont la nature pourrait paraître essentiellement périphérique sont pourtant aussi des centres au regard de certaines des fonctions qu'ils exercent. Prenons un exemple français, un exemple allemand et un exemple marocain. Considérons d'abord L'Isle-sur-laSorgue, commune de 19000 habitants, périphérique d'Avignon, située à $23 \mathrm{~km}$ à l'est de la capitale de Vaucluse. Une partie de son économie est de nature résidentielle avec des actifs qui y habitent tout en se rendant chaque jour sur leur lieu de travail à Avignon. Faut-il en conclure que cette ville se caractérise essentiellement, voire exclusivement, par son caractère périphérique d'Avignon ? Nullement, car elle est aussi un centre à rayonnement international et même intercontinental chaque année, à Pâques et au 15 Août, lors de la Foire Internationale de L'Isle-sur-la-Sorgue. Accueillant des chineurs du monde entier lors de ces foires annuelles, elle se positionne alors à la troisième place européenne du commerce des antiquités et de la brocante après Londres et Saint-Ouen.

Traversons le Rhin pour aller à Walldorf, ville allemande de 15000 habitants située au nord-ouest du Bade-Wurtemberg. A priori, la nature de ses activités pourrait tenir à son caractère périphérique de Karlsruhe, la capitale du Land, située à 37 kilomètres au sud, et de Mannheim, à $24 \mathrm{~km}$ au nord-ouest. Or la réalité est autre. Walldorf est le siège social de l'entreprise $S A P A G$, l'un des plus importants fournisseurs mondiaux de logiciels qui a été créé dans cette ville. Et Walldorf passe pour une des villes les plus riches d'Europe ; elle a été distinguée plusieurs fois comme municipalité d'Allemagne la plus attractive économiquement.

Troisième exemple, Ouarzazate, au Maroc, ville d'environ 100000 habitants du sud marocain, est incontestablement en situation périphérique : périphérie de Rabat où se prennent les grandes décisions politiques concernant le territoire marocain, périphérie de Casablanca, capitale économique du Maroc, périphérie de la France dans la mesure où son attraction touristique dépend notamment du choix d'une clientèle française de choisir cette ville comme lieu ou tête de réseau touristique, périphérie aussi de Bruxelles dans la mesure où le Maroc dispose d'un statut avancé dans le cadre de ses relations avec l'Union européenne. Pourtant, et en même temps, Ouarzazate est un centre international au titre de l'industrie du cinéma; dans ce cadre, elle dispose notamment à proximité du site d'Aït-Ben-Haddou, vil- 
lage inscrit au patrimoine de l'humanité, de paysages remarquables et surtout d'importants studios. Ouarzazate est devenue un haut lieu du cinéma depuis la réalisation de Lawrence d'Arabie en 1962, suivie du tournage d'une partie de nombreux autres films, comme Gladiator (2000) ou Astérix et Obélix : Mission Cléopâtre (2002). En outre, Ouarzazate abrite un musée du cinéma, où sont exposés les décors et costumes ayant servi pour des films tournés dans ses studios.

Une quatrième raison conduit à considérer le modèle centre-périphérie comme désuet : des territoires de nature à dominante pluripériphérique peuvent être centre à une période de l'année. Pensons à Cannes. Cette ville se trouve périphérique d'autres territoires dont les décisions influencent son activité économique, comme celui du siège social de Thales, principal actionnaire d'une des principales entreprises cannoises, Thales Alenia Space. Mais elle est le centre mondial de l'industrie cinématographique chaque année en mai au moment de son Festival.

Au centre de la Bretagne, considérons une petite ville de quelques milliers d'habitants relativement enclavée, située par la voie routière à $85 \mathrm{~km}$ de Brest, et loin de tous les chefs-lieux de département de la Bretagne, à $70 \mathrm{~km}$ de Quimper, à $82 \mathrm{~km}$ de Saint-Brieuc, à $119 \mathrm{~km}$ de Vannes et à $184 \mathrm{~km}$ de Rennes. Elle s'appelle Carhaix-Plouguer dans le Finistère. En 1991, il est décidé d'y créer, géré par des bénévoles, un festival des Vieilles Charrues (Gouel an Erer Kozh en breton). Ce dernier est devenu le plus grand festival de musique français en termes de fréquentation. Chaque été, pendant quelques jours de juillet, Carhaix-Plouguer est donc un centre artistique extrêmement important accueillant plusieurs centaines de milliers de personnes.

Plus généralement, le caractère désuet du modèle centre-périphérie tient au nouveau contexte des territoires tels qu'il résulte des trois dimensions normative, géographique et pratique de la mondialisation au sens large, dimensions qui se traduisent pas les processus de "globalisation ", $\mathrm{d}^{\mathrm{d}}$ " internationalisation " et de mondialisation au sens étroit ${ }^{35}$.

Quant à l'innovation, s'il est vrai que des effets de synergie liés à la proximité entre universités, entreprises et centres de recherche peuvent être intéressants, ils ne sont nullement exclusifs. L'innovation tient davantage à l'esprit d'entrepreneuriat qu'à une localisation dans un centre, ce qui explique les multiples innovations surgissant hors des espaces centraux.

35 Cf. notamment Gérard-François Dumont, « Globalisation, internationalisation, mondialisation : des concepts à clarifier ", Géostratégiques, n², février 2001 ; " La mondialisation et le développement local ", in Alban d’Entremont, Maria Ángeles Lizarraga Lezáun, Juan José Pons Izquierdo, Lucio (Edotires) Recalde Zaratiegui, Homenaje a Manuel Ferrer Regales, Eunsa, Pamplona, 2002 ; "Territoires et potentialités de développement ", Relief, n41, février 2013 ; " Les territoires dans la "mondialisation" : sur un trépied ", Population \& Avenir, n721, janvier-février 2015. 
Le modèle centre-périphérie, sur lequel la théorie géographique s'est longtemps fondée, est souvent promu et mis en application dans des réglementations. Il conduit à penser les territoires sous une analyse duale, distinguant les territoires centre et les territoires périphériques; chacun devait avoir des activités liées à ce positionnement. Selon ce modèle, le territoire-centre n'aurait qu’à exercer au mieux son rôle primatial sur l'ensemble de ses territoires périphériques. Quant à ces derniers, il leur suffirait de s'employer à tirer avantage de leur proximité avec un centre qui les domine ou de se résigner à subir leur éloignement par rapport à celui-ci. Or, ce modèle centrepériphérie est désuet pour de multiples raisons.

En conséquence, aucune organisation administrative des territoires ne peut correspondre à la réalité réticulaire, et donc complexe, des espaces vécus. Plutôt que de courir, comme la France le fait depuis les années $1990^{36}$, après un meccano institutionnel imposé de Paris et obsolète avant même sa conception réglementaire, il aurait été et serait préférable de laisser les territoires libres de choisir leurs liens réticulaires dans l'intérêt du bien commun de leurs habitants.

Enfin, il faut écarter l'idée jacobine selon laquelle toute politique d'aménagement du territoire doit être conçue, à toutes les échelles, de façon centralisée. C'est une erreur de vouloir fonder l'intercommunalité sur un ensemble de compétences systématiquement transférées à un centre : le risque est de corseter tous les territoires qui ont font partie et d'omettre les logiques de subsidiarité, selon lesquelles il ne faut assumer à l'échelon supérieur que tout ce qui ne peut être mieux satisfait à un échelon inférieur.

36 À travers plus de 20 lois. 